\title{
Measuring the Grain Boundary Character and Energy Distributions of Ceramics From Serial Sections of Orientation Maps
}

\author{
Shen J. Dillon* and Gregory S. Rohrer ${ }^{* *}$ \\ *Currently at Department of Materials Science and Engineering, University of Illinois at Urbana- \\ Champaign, 1304 West Green Street, Urbana IL 61801 \\ ** Department of Materials Science and Engineering, Carnegie Mellon University, 5000 Forbes \\ Avenue, Pittsburgh, PA 15213-3890
}

The relative distribution of grain boundaries of different character in a polycrystal and their corresponding energy are important parameters in defining its properties [1]. Directly determining the grain boundary character distribution (GBCD) and the grain boundary energy distribution (GBED) requires knowledge of a material's three-dimensional structure. The GBCD is defined as the relative areas of grain boundaries with distinct grain boundary plane normals and misorientations, and may be defined in units of multiples of a random distribution (MRD) [2]. The GBED describes the relative energies of these boundaries, and can be expressed in relative arbitrary units (a.u.), or units of energy if a reference value exists. Recently, the dual-beam focused ion beam (FIB) scanning electron microscope (SEM) has allowed for automated collection of serial sections of electron backscatter diffraction (EBSD) data $[3,4]$. This presentation will show how the GBCD and GBED may be derived from these serial sections.

Polycrystalline strontium titanate $\left(\mathrm{SrTiO}_{3}\right)(\mathrm{STO})$, was polished to produce a $90^{\circ}$ edge running along one side. The sample analysis was performed along this edge. The sample was sputter-coated with Pt to prevent charging, and attached to a $45^{\circ}$ SEM pre-tilt stub. In this position the sample may be tilted $7^{\circ}-52^{\circ}$ towards the ion-column for ion-milling, or rotated $180^{\circ}$ and tilted $25^{\circ}-70^{\circ}$ towards the EBSD detector for orientation mapping (Nova 600, FEI company, Hillsboro, Or). Samples were ion milled at $30 \mathrm{kV}$ and $3 \mathrm{nA}$ using a $\mathrm{Ga}^{+}$ion beam. Electron backscattered diffraction data was acquired at $20 \mathrm{kV}$ and $9.5 \mathrm{nA}$. Consecutive orientation maps of $\sim 60 \times 95 \mu \mathrm{m}$ at a resolution of 0.3 $\mu \mathrm{m}$, were recorded with a layer spacing of $0.3 \mu \mathrm{m}$.

The orientation maps were aligned using commercially available software (TexSEM, EDAX, Mahwah, NJ) that utilizes a cross-correlation algorithm that analyzes three color channels in the maps. The reconstructed image is shown in Figure 1. The commercial software was also used to extract grain boundary line segments in each layer, with a maximum deviation of two pixels. Triple lines were determined by comparing neighboring junctions in each layer and matching junctions whose surrounding grains had misorientations less than $5^{\circ}$. These triple lines are utilized to perform a sub-pixel alignment, based on the assumed random distribution of triple line directions. Triple lines were defined across alternate layers to minimize errors. The cross-product of the triple junction line defines the grain boundary plane normal, and half of the cross product gives the area. These parameters may be used to describe the GBCD. The grain boundary plane normals and triple line vectors are also analyzed to solve for the GBED based on the Herring condition [5], using the procedure developed by Moraweic [6].

Figure 2 compares the grain boundary plane normals from the GBCD and GBED. There is an inverse relationship between these two parameters that occurs as the system attempts to minimize its 
energy. These results are in agreement with previous results for STO based on stereology and surface energy analysis; although the dynamic range of the anisotropy is reduced [7].

References

[1] G.S. Rohrer, D.M. Saylor, B.S. El-Dasher, B.L. Adams, A.D. Rollett, and P. Wynblatt, Z. Metall., 95 (2004) 214.

[2] C.-S. Kim, A.D. Rollett, G.S. Rohrer, Scripta Mater. 54 (2006) 1005.

[3] J. Konrad, S. Zaefferer, and D. Raabe, Acta Mater. 54 (2006) 1369.

[4] M. D. Uchic, M. A. Groeber, D. M. Dimiduk, and J. P. Simmons, Scripta Mater. 55 (2006) 23.

[5] C. Herring, Phys. Rev. 82 (1951) 87.

[6] A. Morawiec, Acta Mater. 48 (2000) 3525.

[7] D.M. Saylor, B.S. El-Dasher, T. Sano, and G.S. Rohrer, J. Amer. Ceram. Soc. 87 (2004) 670. Support from the MRSEC program of the NSF under Award DMR-0520425 is acknowledged.

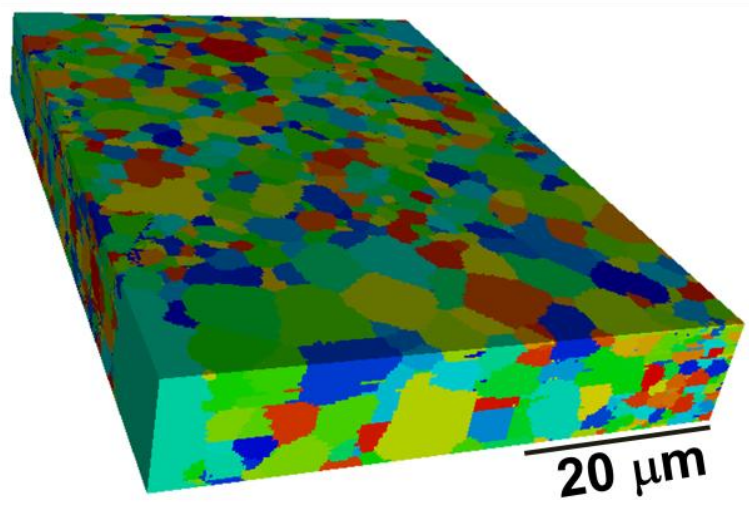

Fig. 1. Reconstruction of 40 serial sections of EBSD data collected from STO.

\section{(a) MRD}

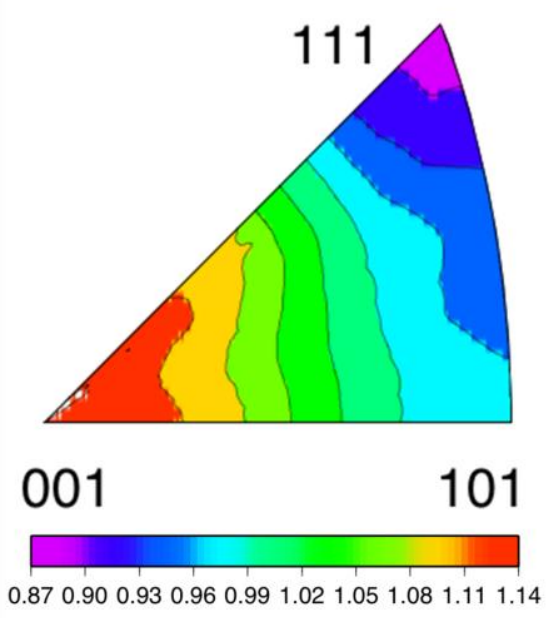

(b) a.u.

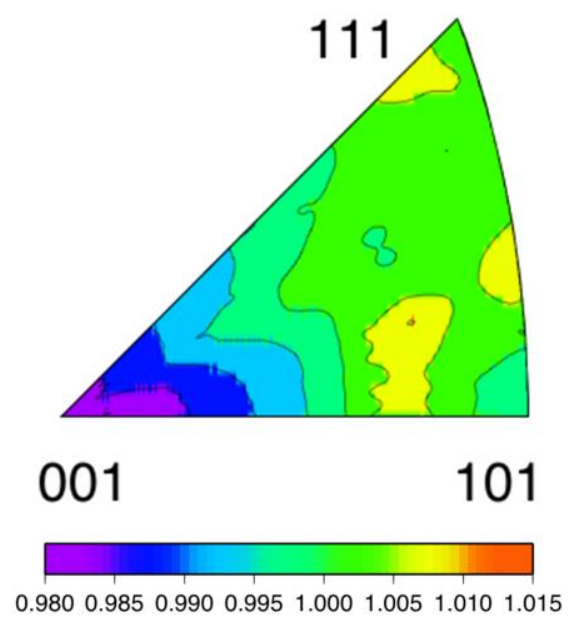

Fig. 2. (a) The distribution of grain boundary plane normals of STO expressed in MRD and (b) the corresponding average relative energies of those planes. 